\title{
Topological structuring of the digital plane
}

\author{
Josef Šlapal ${ }^{\dagger}$ \\ Institute of Mathematics, Brno University of Technology, 61669 Brno, Czech Republic
}

received $24^{\text {th }}$ October 2011, revised $14^{\text {th }}$ July 2013, accepted $24^{\text {th }}$ July 2013.

We discuss an Alexandroff topology on $\mathbb{Z}^{2}$ having the property that its quotient topologies include the Khalimsky and Marcus-Wyse topologies. We introduce a further quotient topology and prove a Jordan curve theorem for it.

Keywords: digital plane, quotient topology, Khalimsky topology, Jordan curve

\section{Introduction}

In the classical approach to digital topology (see e.g. [12] and [13]), graph theoretic tools are used for structuring $\mathbb{Z}^{2}$, namely the well-known binary relations of 4-adjacency and 8-adjacency. But neither 4adjacency nor 8-adjacency itself allows an analogue of the Jordan curve theorem (cf. [9]) and, therefore, one has to use a combination of the two adjacencies. To overcome this disadvantage, a new, purely topological approach to the problem was proposed in [6] which utilizes a convenient topology on $\mathbb{Z}^{2}$, called the Khalimsky topology (cf. [5]), for structuring the digital plane. At present, this topology is one of the most important concepts of digital topology. It has been studied and used by many authors, see e.g. [3] and [7]-[10].

The possibility of employing convenient topological structures on $\mathbb{Z}^{2}$ different from the Khalimsky topology is discussed in [14]-[19]. Particularly, in [16], a new topology on $\mathbb{Z}^{2}$ is introduced and it is shown there that this topology provides certain convenient Jordan curves behaving more advantageously than the Jordan curves in the Khalimsky space. The quotient topologies of the topology from [16] are studied in [17] where it is shown that they include, among others, the Khalimsky and Marcus-Wyse topologies. In the present note we continue the investigations from [16] and [17]. We discuss a topology on $\mathbb{Z}^{2}$ which is finer than the topology introduced in [16] but still has the property that the Khalimsky and Marcus-Wyse topologies belong to its quotient topologies. We study another of its quotient topologies on $\mathbb{Z}^{2}$, denoted by $v$, and prove a Jordan curve theorem for it. This Jordan curve theorem differs from the Jordan curve theorems for the Euclidean plane, the Khalimsky plane, and the $(4,8)$ and $(8,4)$ digital planes of "classical" digital topology in the following significant ways:

(i) The paper's Jordan curve theorem only applies to certain simple closed curves in $\left(\mathbb{Z}^{2}, v\right)$. There are arbitrarily long simple closed curves $C$ in $\left(\mathbb{Z}^{2}, v\right)$ for which $\mathbb{Z}^{2} \backslash C$ has more than two components.

\footnotetext{
${ }^{\dagger}$ Email: slapalefme.vutbr.cz. This work was supported by the Brno University of Technology, projects no. FSI-S-11-3 and FEKT/FSI-S-11-1.

1365-8050 (c) 2013 Discrete Mathematics and Theoretical Computer Science (DMTCS), Nancy, France
} 
(ii) Those simple closed curves $C$ in $\left(\mathbb{Z}^{2}, v\right)$ to which the paper's Jordan curve theorem applies will in most cases not be the common boundary of the two components of $\mathbb{Z}^{2} \backslash C$.

\section{Preliminaries}

For the topological terminology used we refer to [2] and [4]. Throughout the note, all topologies dealt with are thought of as being (given by) Kuratowski closure operators. Recall that a topology $p$ on a set $X$ is said to be a $T_{\frac{1}{2}}$-topology if each singleton subset of $X$ is closed or open (so that $T_{\frac{1}{2}}$ implies $T_{0}$ ), and it is called an Alexandroff topology if $p A=\bigcup_{x \in A} p\{x\}$ whenever $A \subseteq X$. A pair $(X, p)$ where $X$ is a set and $p$ is an Alexandroff topology on $X$ is called an Alexandroff space. Recall also that, given topological spaces $(X, p)$ and $(Y, q)$, a surjection $e: X \rightarrow Y$ is said to have connected fibres if $e^{-1}(\{y\})$ is connected (in $(X, p)$ ) for every point $y \in Y$.

If we omit, in the definition of Kuratowski closure operators (Kuratowski closure spaces), the requirement of idempotency, we obtain the so-called pretopologies (pretopological spaces) - cf. [2]. We will use some concepts naturally extended from topologies (topological spaces) to pretopologies (pretopological spaces). Given pretopologies $p_{1}, p_{2}$ on a set $X$, we say that $p_{1}$ is finer that $p_{2}$ if $p_{1}(A) \subseteq p_{2}(A)$ for every subset $A \subseteq X$. A map $f:(X, p) \rightarrow(Y, q)$, where $(X, p)$ and $(Y, q)$ are pretopological spaces, is called continuous if $f(p A) \subseteq q(f(A))$ for every $A \subseteq X$. Let $e: X \rightarrow Y$ be a surjection and $p$ be a pretopology on $X$. Then the finest pretopology $q$ on $Y$ such that $e:(X, p) \rightarrow(Y, q)$ is continuous is called the quotient pretopology of $p$ generated by $e$.

Of course, if a quotient pretopology of a topology $p$ (generated by a surjection $e$ ) is a topology, then it is a quotient topology of $p$ (generated by $e$ ). The proof of our main theorem will be based on a result (Proposition 4.1) about such quotient topologies of Alexandroff topologies.

By a graph on a set $V$ we always mean an undirected simple graph without loops with the vertex set $V$. Recall that a path in a graph is a finite (nonempty) sequence $x_{0}, x_{1}, \ldots, x_{n}$ of pairwise different vertices such that $x_{i-1}$ and $x_{i}$ are adjacent (i.e., joined by an edge) whenever $i \in\{1,2, \ldots n\}$. Here, $x_{0}$ and $x_{n}$ are said to be the end vertices of the path. By a cycle in a graph we understand any finite set of at least three vertices which can be ordered into a path with the end vertices adjacent.

The connectedness graph of a topology $p$ on $X$ is the graph on $X$ in which a pair of vertices $x, y$ are adjacent if and only if $x \neq y$ and $\{x, y\}$ is a connected subset of $(X, p)$. Let $p$ be an Alexandroff topology on a set $X$. Then it is obvious that a subset $A \subseteq X$ is connected in $(X, p)$ if and only if each pair of points of $A$ may be joined by a path in the connectedness graph of $(X, p)$ contained in $A$. In the sequel, only connectedness graphs of connected Alexandroff topologies on $\mathbb{Z}^{2}$ will be displayed. In these graphs, the closed points will be ringed and the mixed ones (i.e., the points that are neither closed nor open) boxed. Hence, the points neither ringed nor boxed will be open (note that no points of $\mathbb{Z}^{2}$ may be both closed and open).

By a (discrete) closed curve in a topological space $(X, p)$ we mean a cycle in the connectedness graph of $p$. Thus, every closed curve is a nonempty, finite and connected set. In accordance with [16], a closed curve $C \subseteq X$ in $(X, p)$ is said to be simple if, for each point $x \in C$, there are exactly two points of $C$ adjacent to $x$ in the connectedness graph of $p$. A simple closed curve $C$ in $(X, p)$ is said to be a (discrete) Jordan curve if it separates $(X, p)$ into precisely two components (i.e., if the subspace $X \backslash C$ of $(X, p)$ consists of precisely two components).

We will need the following statement resulting from [15], Corollary 1.5: 
Lemma 2.1 Let $(X, p),(Y, q)$ be Alexandroff spaces such that $q$ is the quotient pretopology of penerated by a surjection $e: X \rightarrow Y$ with connected fibres. Then a subset $B \subseteq Y$ is connected in $(Y, q)$ if and only if $e^{-1}(B)$ is connected in $(X, p)$.

Note that the statement of the previous Lemma need not be true if $q$ is only the quotient topology and not the quotient pretopology of $p$ - see the following counterexample due to one of the referees of this paper:

Example 2.2 Let $(X, p)$ be the topological space where $X=\{1,2,3,4\}$, the closure of $\{1\}$ is $\{1\}$, the closure of $\{2\}$ is $\{1,2,3\}$, the closure of $\{3\}$ is $\{3\}$, and the closure of $\{4\}$ is $\{3,4\}$. Let $Y=\{a, b, c\}$ and let $e: X \rightarrow Y$ be the surjection defined by $e(1)=a, e(2)=e(3)=b$, and $e(4)=c$ (so that $e$ has connected fibers). Then, in the quotient space $(Y, q)$ of $(X, p)$ generated by $e$, we have that $a$ lies in the closure of $c$. So, $B=\{a, c\}$ is connected in $(Y, q)$, but $e^{-1}(B)=\{1,4\}$ is not connected in $(X, p)$.

Let $z=(x, y) \in \mathbb{Z}^{2}$ be a point. We put

$H_{2}(z)=\{(x+k, y) ; k \in\{-1,1\}\}$,

$V_{2}(z)=\{(x, y+l) ; l \in\{-1,1\}\}$,

$D_{5}(z)=H_{2}(z) \cup\{(x+k, y-1) ; k \in\{-1,0,1\}\}$,

$U_{5}(z)=H_{2}(z) \cup\{(x+k, y+1) ; k \in\{-1,0,1\}\}$

$L_{5}(z)=V_{2}(z) \cup\{(x-1, y+l) ; l \in\{-1,0,1\}\}$,

$R_{5}(z)=V_{2}(z) \cup\{(x+1, y+l) ; l \in\{-1,0,1\}\}$,

$A_{12}(z)=\left\{(k, l) \in \mathbb{Z}^{2} ; k=x\right.$ and $0<|l-y| \leq 3$, or $l=y$ and $\left.0<|k-x| \leq 3\right\}$.

Next, we put

$A_{4}(z)=H_{2}(z) \cup V_{2}(z)$,

$A_{8}(z)=L_{5}(z) \cup R_{5}(z)\left(=D_{5}(z) \cup U_{5}(z)\right)$, and

$A_{4}^{\prime}(z)=A_{8}(z) \backslash A_{4}(z)$.

Thus, the number of points of each of the nine sets introduced above equals the index of the symbol denoting this set. In the literature, the points of $A_{4}(z)$ and $A_{8}(z)$ are said to be 4-adjacent and 8-adjacent to $z$, respectively. It is natural to call the points of $H_{2}(z), V_{2}(z), D_{5}(z), U_{5}(z), L_{5}(z), R_{5}(z), A_{12}(z)$ and $A_{4}^{\prime}(z)$ horizontally 2-adjacent, vertically 2-adjacent, down 5-adjacent, up 5-adjacent, left 5-adjacent, right 5-adjacent, cross 12-adjacent and diagonally 4-adjacent to $z$, respectively. Clearly, each of these adjacencies, excluding $A_{12}(z)$ implies 8-adjacency.

The union of each of the above ten sets $H_{2}(z), V_{2}(z) \ldots$ with the singleton $\{z\}$ is denoted by the corresponding barred symbols, i.e., by $\bar{H}_{2}(z), \bar{V}_{2}(z) \ldots$.

Recall [6] that the Khalimsky topology on $\mathbb{Z}^{2}$ is the Alexandroff topology $t$ given as follows:

For any $z=(x, y) \in \mathbb{Z}^{2}$,

$$
t\{z\}=\left\{\begin{array}{l}
\bar{A}_{8}(z) \text { if } x, y \text { are even } \\
\bar{H}_{2}(z) \text { if } x \text { is even and } y \text { is odd } \\
\bar{V}_{2}(z) \text { if } x \text { is odd and } y \text { is even } \\
\{z\} \text { otherwise. }
\end{array}\right.
$$

The Khalimsky topology is connected and $T_{0}$; a portion of its connectedness graph is shown in Figure 1.

In [6], Khalimsky, Koppermann and Meyer proved the following digital Jordan curve theorem for $\left(\mathbb{Z}^{2}, t\right)$ : 


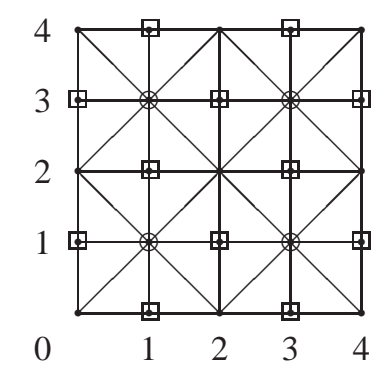

Fig. 1: A portion of the connectedness graph of the Khalimsky topology.

Theorem 2.3 In the Khalimsky plane, any simple closed curve having at least four points is a Jordan curve.

The Marcus-Wyse topology (cf. [11]) is the connected Alexandroff $T_{\frac{1}{2}}$-topology $s$ on $\mathbb{Z}^{2}$ given as follows:

For any $z=(x, y) \in \mathbb{Z}^{2}$,

$$
s\{z\}=\left\{\begin{array}{l}
\bar{A}_{4}(z) \text { if } x+y \text { is odd } \\
\{z\} \text { otherwise. }
\end{array}\right.
$$

The Marcus-Wyse topology is quite simple - its connectedness graph coincides with the 4-adjacency graph. The following digital Jordan curve theorem for $\left(\mathbb{Z}^{2}, s\right)$ is proved in [8]:

Theorem 2.4 Let $C$ be a cycle in the connectedness graph of $s$ such that, for every point $z \in C$, there are precisely two points of $C$ adjacent to $z$ and $\operatorname{card}\left\{p \in C \backslash\{z\}\right.$; there exists a point $q \in \mathbb{Z}^{2}$ adjacent to both $z$ and $p\}=2$. Then $C$ is a Jordan curve in $\left(\mathbb{Z}^{2}, s\right)$.

The previous Theorem has been recently generalized to a larger class of curves in [1].

Note that, at none of its points, can a Jordan curve in the Khalimsky or Marcus-Wyse plane turn to make an acute angle of $\frac{\pi}{4}$ (and, in mixed points, a Jordan curve in the Khalimsky plane can not turn at all). In the next section, we will introduce a topology on $\mathbb{Z}^{2}$ with respect to which Jordan curves may turn, at some points, to make an acute angle of $\frac{\pi}{4}$.

\section{Topology $w$ and some of its quotients}

We denote by $w$ the Alexandroff topology on $\mathbb{Z}^{2}$ given as follows (cf. [18]):

For any point $z=(x, y) \in \mathbb{Z}^{2}$,

$$
w\{z\}=\left\{\begin{array}{l}
\bar{A}_{8}(z) \text { if } x=4 k, y=4 l, k, l \in \mathbb{Z}, \\
\bar{A}_{4}^{\prime}(z) \text { if } x=2+4 k, y=2+4 l, k, l \in \mathbb{Z}, \\
\bar{D}_{5}(z) \text { if } x=2+4 k, y=1+4 l, k, l \in \mathbb{Z}, \\
\bar{U}_{5}(z) \text { if } x=2+4 k, y=3+4 l, k, l \in \mathbb{Z}, \\
\bar{L}_{5}(z) \text { if } x=1+4 k, y=2+4 l, k, l \in \mathbb{Z}, \\
\bar{R}_{5}(z) \text { if } x=3+4 k, y=2+4 l, k, l \in \mathbb{Z}, \\
\bar{H}_{2}(z) \text { if } x=2+4 k, y=4 l, k, l \in \mathbb{Z}, \\
\bar{V}_{2}(z) \text { if } x=4 k, y=2+4 l, k, l \in \mathbb{Z}, \\
\{z\} \text { otherwise. }
\end{array}\right.
$$




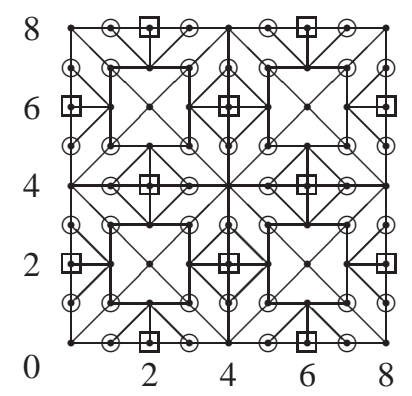

Fig. 2: A portion of the connectedness graph of $w$.

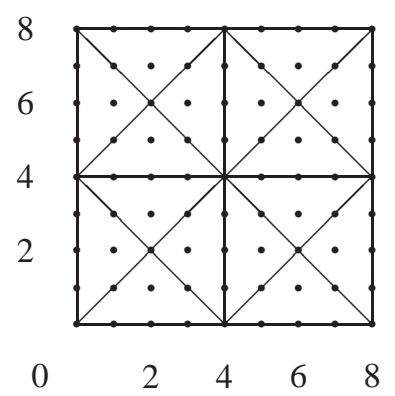

Fig. 3: A portion of a subgraph of the connectedness graph of $w$.

Clearly, $w$ is connected and $T_{0}$. A portion of the connectedness graph of $w$ is shown in Figure 2.

The Alexandroff topology on $\mathbb{Z}^{2}$ introduced and studied in [16] is coarser than $w$ - it is obtained from the definition of $w$ by replacing $\bar{D}_{5}(z), \bar{U}_{5}(z), \bar{L}_{5}(z)$ and $\bar{R}_{5}(z)$ by $\bar{D}_{5}(z) \backslash\{(x, y-1)\}, \bar{U}_{5}(z) \backslash\{(x, y+$ $1)\}, \bar{L}_{5}(z) \backslash\{(x-1, y)\}$ and $\bar{R}_{5}(z) \backslash\{(x+1, y)\}$, respectively. Because of this fact, some results proved in [16] and [17] for the topology studied there may easily be transferred to $w$. In particular, by Theorem 11 in [16], we get:

Theorem 3.1 Every cycle $C$ in the graph a portion of which is shown in Figure 3 is a Jordan curve in $\left(\mathbb{Z}^{2}, w\right)$ having the property that, if $C$ has no acute corners, then for each point $z \in C$ the set $A_{8}(z)$ contains at least one point in each of the components of $\mathbb{Z}^{2} \backslash C$.

Analogously to [17], we define an Alexandroff topology $v$ on $\mathbb{Z}^{2}$ as follows:

For any $z=(x, y) \in \mathbb{Z}^{2}$,

$$
v\{z\}=\left\{\begin{array}{l}
\bar{H}_{2}(z) \text { if } x \text { is odd and } y \text { is even } \\
\bar{V}_{2}(z) \text { if } x \text { is even and } y \text { is odd } \\
\bar{A}_{4}^{\prime}(z) \text { if } x, y \text { are odd } \\
\{z\} \text { if } x, y \text { are even. }
\end{array}\right.
$$




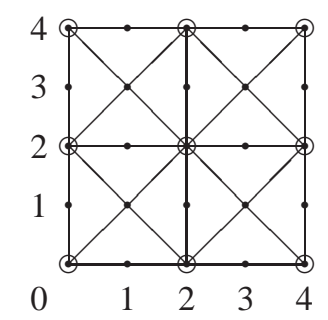

Fig. 4: The connectedness graph of $v$.

Evidently, $v$ is connected and $T_{\frac{1}{2}}$. A portion of its connectedness graph is shown in Figure 4.

The following three statements are obtained by transferring the results of Theorems 10-12 in [17] to the topology $w$ :

Proposition 3.2 The Khalimsky topology is the quotient pretopology of $w$ generated by the surjection $f: \mathbb{Z}^{2} \rightarrow \mathbb{Z}^{2}$ given as follows:

$$
f(x, y)=\left\{\begin{array}{l}
(2 k, 2 l) \text { if }(x, y)=(4 k, 4 l), k, l \in \mathbb{Z}, \\
(2 k, 2 l+1) \text { if }(x, y) \in \bar{A}_{4}(4 k, 4 l+2), k, l \in \mathbb{Z}, \\
(2 k+1,2 l) \text { if }(x, y) \in \bar{A}_{4}(4 k+2,4 l), k, l \in \mathbb{Z}, \\
(2 k+1,2 l+1) \text { if }(x, y) \in \bar{A}_{4}^{\prime}(4 k+2,4 l+2), k, l \in \mathbb{Z} .
\end{array}\right.
$$

The surjection $f$ is shown in Figure 5 where the corresponding decomposition of the topological space $\left(\mathbb{Z}^{2}, w\right)$ is marked by the dashed lines. All points of a class of the decomposition are mapped by $f$ to the center point of the class expressed in the bold coordinates.

Proposition 3.3 The Marcus-Wyse topology s is the quotient pretopology of $w$ generated by the surjection $g: \mathbb{Z}^{2} \rightarrow \mathbb{Z}^{2}$ given as follows:

$$
g(x, y)=\left\{\begin{array}{c}
(k+l, l-k) \text { if }(x, y) \in \bar{A}_{8}(4 k, 4 l), k, l \in \mathbb{Z}, \\
(k+l+1, l-k) \text { if }(x, y)=(4 k+2,4 l+2) \text { for some } k, l \in \mathbb{Z} \\
\quad \text { with } k+l \text { odd or }(x, y) \in \bar{A}_{12}(4 k+2,4 l+2) \text { for some } \\
\quad k, l \in \mathbb{Z} \text { with } k+l \text { even } .
\end{array}\right.
$$

The decomposition of the topological space $\left(\mathbb{Z}^{2}, w\right)$ given by $g$ is shown in Figure 6, similarly to Figure 5 , by the dashed lines. Every class of the decomposition is mapped by $g$ to its center point expressed in the coordinates with respect to the diagonal axes (where the first coordinate relates to the axis with only the non-negative part displayed).

Proposition 3.4 $v$ is the quotient pretopology of $w$ generated by the surjection $h: \mathbb{Z}^{2} \rightarrow \mathbb{Z}^{2}$ given as follows:

$$
h(x, y)=\left\{\begin{array}{l}
(2 k, 2 l) \text { if }(x, y) \in \bar{A}_{8}(4 k, 4 l), k, l \in \mathbb{Z}, \\
(2 k, 2 l+1) \text { if }(x, y) \in \bar{H}_{2}(4 k, 4 l+2), k, l \in \mathbb{Z}, \\
(2 k+1,2 l) \text { if }(x, y) \in \bar{V}_{2}(4 k+2,4 l), k, l \in \mathbb{Z}, \\
(2 k+1,2 l+1) \text { if }(x, y)=(4 k+2,4 l+2), k, l \in \mathbb{Z} .
\end{array}\right.
$$

The surjection $h$ is demonstrated in Figure 7 in a way analogous to that used to demonstrate $f$ in Figure 5 . 


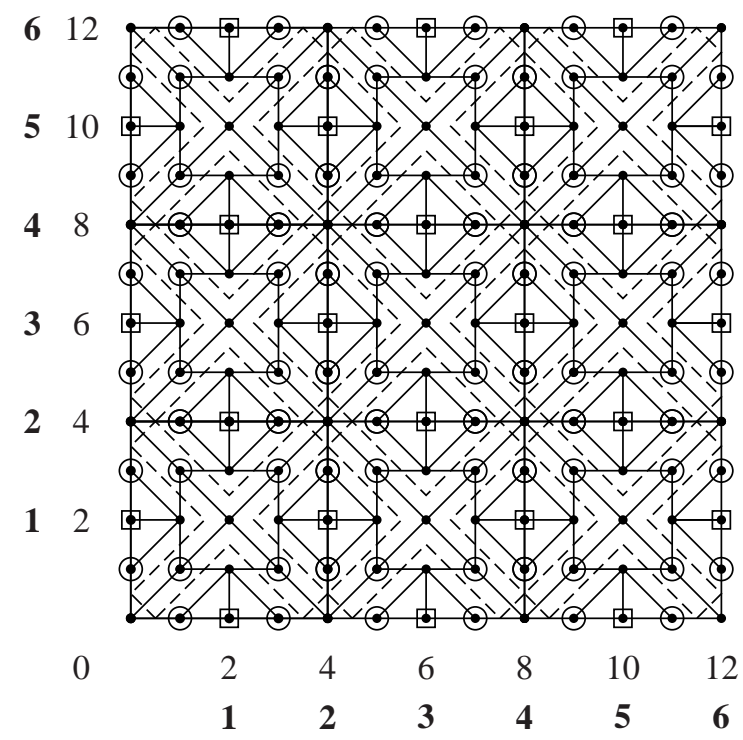

Fig. 5: Decomposition of $\left(\mathbb{Z}^{2}, w\right)$ given by the surjection $f$.

\section{Jordan curves in $\left(\mathbb{Z}^{2}, v\right)$}

In Theorem 3.1, we have determined Jordan curves in the topological space $\left(\mathbb{Z}^{2}, w\right)$. The following statement enables us to use Theorem 3.1 to determine Jordan curves in a quotient topological space of $\left(\mathbb{Z}^{2}, w\right)$ :

Proposition 4.1 Let $\left(\mathbb{Z}^{2}, p\right),\left(\mathbb{Z}^{2}, q\right)$ be Alexandroff spaces such that $q$ is the quotient pretopology of $p$ on $\mathbb{Z}^{2}$ generated by a surjection e $: \mathbb{Z}^{2} \rightarrow \mathbb{Z}^{2}$. Let e have connected fibres and let $D \subseteq \mathbb{Z}^{2}$ be a simple closed curve in $\left(\mathbb{Z}^{2}, q\right)$. Then $D$ is a Jordan curve in $\left(\mathbb{Z}^{2}, q\right)$ if the following two conditions are satisfied:

(1) There is a Jordan curve $C$ in $\left(\mathbb{Z}^{2}, p\right)$ such that $e(C)=D$.

(2) $C_{i} \backslash e^{-1}(D)$ is nonempty and connected in $\left(\mathbb{Z}^{2}, p\right)$ for $i=1,2$ where $C_{1}$ and $C_{2}$ are the two components of $\mathbb{Z}^{2} \backslash C$.

Proof: Let the conditions of the statement be fulfilled and put $C_{1}^{\prime}=C_{1} \backslash e^{-1}(D)$ and $C_{2}^{\prime}=C_{2} \backslash e^{-1}(D)$. Since $C_{i}^{\prime}$ is connected for $i=1,2$ (by the assumptions), $e\left(C_{i}^{\prime}\right)$ is connected for $i=1,2$ by Lemma 2.1. On the other hand, since $C_{1}^{\prime} \cup C_{2}^{\prime}=e^{-1}\left(\mathbb{Z}^{2} \backslash D\right)$ is not connected (by the assumptions), $\mathbb{Z}^{2} \backslash D=$ $e\left(C_{1}^{\prime}\right) \cup e\left(C_{2}^{\prime}\right)$ is not connected by Lemma 2.1. Hence, $e\left(C_{1}^{\prime}\right)$ and $e\left(C_{2}^{\prime}\right)$ are the components of $\mathbb{Z}^{2} \backslash D$.

Indeed, Proposition 4.1 may be applied to get a Jordan curve theorem in the spaces $\left(\mathbb{Z}^{2}, t\right)$ and $\left(\mathbb{Z}^{2}, v\right)$ because the maps $f$ and $h$ have connected fibres. This is not true if $w$ is replaced by the topology studied in [16] (because then only $f$ has connected fibres). The map $g$ has connected fibres neither for $w$ nor for the topology from [16]. 


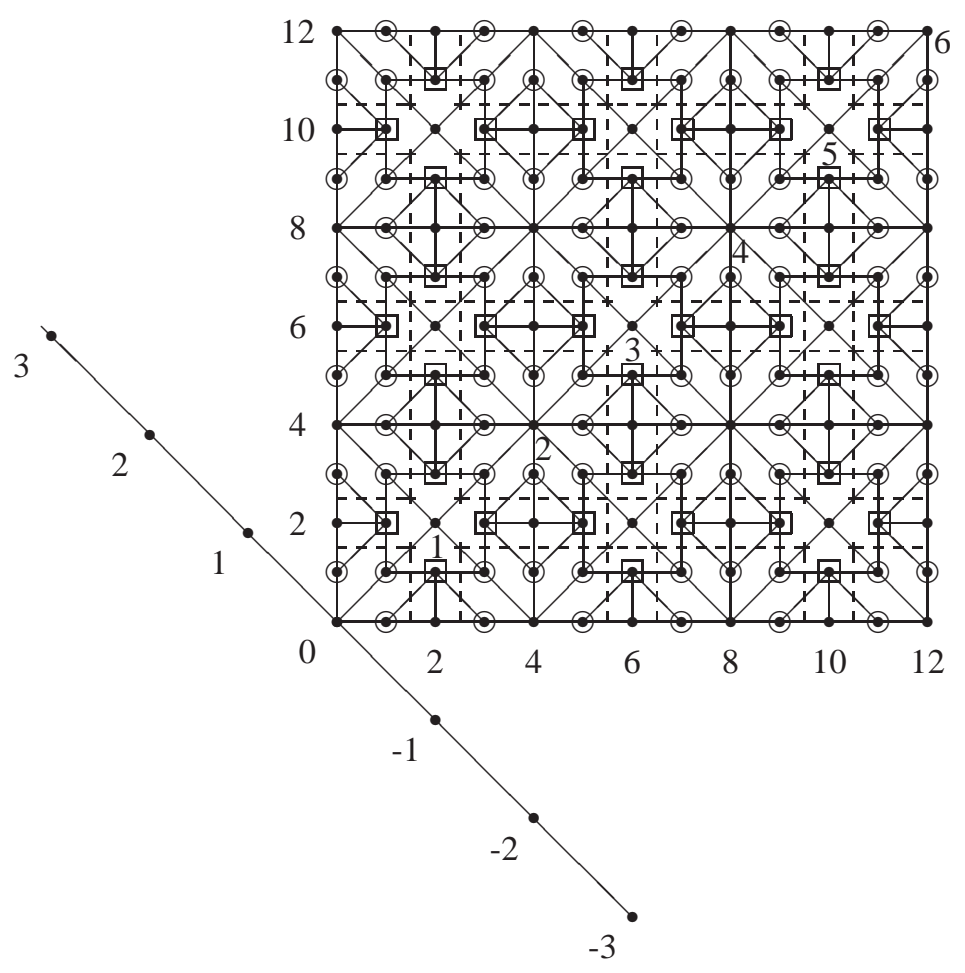

Fig. 6: Decomposition of $\left(\mathbb{Z}^{2}, w\right)$ given by the surjection $g$. 


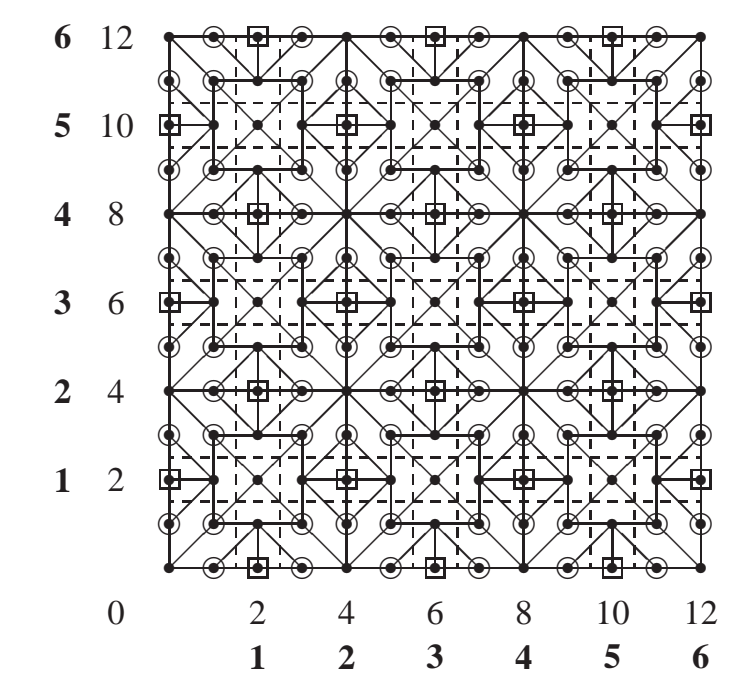

Fig. 7: Decomposition of $\left(\mathbb{Z}^{2}, w\right)$ given by the surjection $h$.

As for the Khalimsky topology, we have the famous Theorem 2.3 proved in [6] that completely determines Jordan curves among the simple closed ones. For the Marcus-Wyse topology, we have Theorem 2.4 and also Jordan curve theorem proved in [16] (Proposition 13). Therefore, as the main result of this note, we will prove a Jordan curve theorem for $v$.

Theorem 4.2 Let $D$ be a simple closed curve in $\left(\mathbb{Z}^{2}, v\right)$ with more than four points such that every pair of different points $z_{1}, z_{2} \in D$ with both coordinates even satisfies $A_{4}\left(z_{1}\right) \cap A_{4}\left(z_{2}\right) \subseteq D$. Then $D$ is a Jordan curve in $\left(\mathbb{Z}^{2}, v\right)$.

Proof: By Proposition 3.3, $v$ is the quotient pretopology of $w$ generated by $h$. It may easily be seen that, in the graph a portion of which is shown in Fig. 3, there is precisely one cycle $C$ that satisfies $h(C)=D$. By Theorem 3.1, $C$ is a Jordan curve in $\left(\mathbb{Z}^{2}, w\right)$. Clearly, every point of $C$ has either two even coordinates or two odd coordinates or a coordinate that is a multiple of four. $C$ consists of the center points of the sets $h^{-1}(z), z \in C$, (see Fig. 7) and the points lying between the pairs of center points of the sets $h^{-1}\left(z_{1}\right)$ and $h^{-1}\left(z_{2}\right)$ where $z_{1}, z_{2} \in D$ are adjacent points in the connectedness graph of $v$ (clearly, for every pair of points $z_{1}, z_{2} \in D$ adjacent in the connectedness graph of $v$, there is precisely one point lying between the center point of $h^{-1}\left(z_{1}\right)$ and that of $h^{-1}\left(z_{2}\right)$ - it is the only point adjacent to each of the two center points in the graph shown in Fig. 2). Since $D$ is a simple closed curve in $\left(\mathbb{Z}^{2}, v\right)$, we have $\operatorname{card}(\{(x+i, y+j) ; i, j \in\{-2,2\}\} \cap C)=2$ for every point $(x, y) \in C$ with $x=4 k+2$ and $y=4 l+2$ for some $k, l \in \mathbb{Z}$. Further, we have card $C>8$ because $C$ has more than four points. The fact that every pair of different points $z_{1}, z_{2} \in h(C)$ with both coordinates even satisfies $A_{4}\left(z_{1}\right) \cap A_{4}\left(z_{2}\right) \subseteq h(C)$ implies that

(a) $(4 k, 4 l+2) \in C$ whenever $(4 k, 4 l),(4 k, 4 l+4) \in C(k, l \in \mathbb{Z})$ and

(b) $(4 k+2,4 l) \in C$ whenever $(4 k, 4 l),(4 k+4,4 l) \in C(k, l \in \mathbb{Z})$. 
Let $C_{1}, C_{2}$ be the two components of the subspace $\mathbb{Z}^{2} \backslash C$ of $\left(\mathbb{Z}^{2}, w\right)$ and put $C_{i}^{\prime}=C_{i} \backslash h^{-1}(D)$ for $i=1,2$. Since $C$ has more than four points, we have $C_{i}^{\prime} \neq \emptyset$ for $i=1,2$. Let $(x, y) \in D$ be a point and write $h^{-1}(x, y)$ briefly instead of $h^{-1}(\{(x, y)\})$. It is evident that $h^{-1}(x, y) \subseteq C$ if and only if both $x$ and $y$ are odd (then $h^{-1}(x, y)$ is a singleton). Thus, let $(x, y) \in D$ be a point with $x$ or $y$ even.

(1) Suppose that $x$ is even and $y$ is odd. Then $h^{-1}(x, y)=\bar{H}_{2}(2 x, 2 y)$ where $(2 x, 2 y) \in C$. The points $h(2 x, 2 y-1)=(x, y-1)$ and $h(2 x, 2 y+1)=(x, y+1)$ must also lie in $D$, so that $(2 x, 2 y-1)$ and $(2 x, 2 y+1)$ both lie in $C$, but $h(2 x-2,2 y)=(x-1, y)$ does not lie in $D$, so that $(2 x-2,2 y) \notin C$. Therefore, every path (in the connectedness graph of $w)$ in $C_{i}(i \in\{1,2\})$ containing the point $(2 x-1,2 y)$ as a non-end vertex contains also the points $(2 x-1,2 y-1)$ and $(2 x-1,2 y+1)$ because they are the only points of $C_{i}$ adjacent to $(2 x-1,2 y)$ in the connectedness graph of $w$. Thus, given any path in $C_{i}$ $(i \in\{1,2\})$ that contains the point $(2 x-1,2 y)$, when we replace the point $(2 x-1,2 y)$ by $(2 x-2,2 y)$, we obtain another path in $C_{i}$ because $(2 x-2,2 y)$ is adjacent to both $(2 x-1,2 y-1)$ and $(2 x-1,2 y+1)$. It follows that $C_{i} \backslash\{(2 x-1,2 y)\}$ is connected for $i \in\{1,2\}$. Using analogous arguments, we may show that $C_{i} \backslash\{(2 x+1,2 y)\}$ is also connected for $i \in\{1,2\}$. Thus, $C_{i} \backslash h^{-1}(x, y)$ is connected for $i=1,2$.

(2) If $x$ is odd and $y$ is even, then $h^{-1}(x, y)=\bar{V}_{2}(2 x, 2 y)$ where $(2 x, 2 y) \in C$ and the situation is analogous to (1), so that $C_{i} \backslash h^{-1}(x, y)$ is connected for $i=1,2$.

Put $D_{0}=\{(m, n) \in D ; m+n$ is odd $\}$ and $C_{i}^{\prime \prime}=C_{i} \backslash h^{-1}\left(D_{0}\right)$. Then (1) and (2) imply that $C_{i}^{\prime \prime}$ is connected for $i=1,2$. We will now deduce from the connectedness of $C_{i}^{\prime \prime}$ that $C_{i} \backslash h^{-1}(D)=$ $C_{i}^{\prime \prime} \backslash h^{-1}(D)$ must also be connected (for $i=1,2$ ). It will then follow from Proposition 4.1 that $D$ is a Jordan curve in $\left(\mathbb{Z}^{2}, v\right)$. In the sequel, the term "path" will mean "path in the connectedness graph of $w$ ".

The proof that $C_{i} \backslash h^{-1}(D)$ is connected is based on the fact that, if $P$ is any path in $C_{i}^{\prime \prime}$ whose first and last points lie in $C_{i} \backslash h^{-1}(D)$, and $z$ is any point of $P$ in $h^{-1}(D)$ (so that $z \in C_{i}^{\prime \prime} \cap h^{-1}(D)$ ), then: (i) The immediate predecessor and the immediate successor of $z$ in the path $P$ both lie in $\mathbb{Z}^{2} \backslash h^{-1}(D)$, and hence in $C_{i} \backslash h^{-1}(D)$.

(ii) There is a short path (of length at most 4) in $C_{i} \backslash h^{-1}(D)$ from the immediate predecessor of $z$ in $P$ to the immediate successor of $z$ in $P$.

Readily, (i) and (ii) imply that $C_{i} \backslash h^{-1}(D)$ is connected: Indeed, if $a$ and $b$ are any points in $C_{i} \backslash h^{-1}(D)=$ $C_{i}^{\prime \prime} \backslash h^{-1}(D)$, then (as $C_{i}^{\prime \prime}$ is connected) there is a path $P$ in $C_{i}^{\prime \prime}$ from $a$ to $b$ and, since we can use the short path referred to in (ii) as a "detour" in $C_{i} \backslash h^{-1}(D)$ around any point $z$ of $P$ that lies in $h^{-1}(D)$, there is also a path in $C_{i} \backslash h^{-1}(D)$ from $a$ to $b$. So, it remains only to confirm that (i) and (ii) are true.

(i) follows from the observation that no two points of $C_{i}^{\prime \prime} \cap h^{-1}(D)$ are adjacent in $w$ 's connectedness graph because each point of $C_{i}^{\prime \prime} \cap h^{-1}(D)$ lies in $A_{8}(c)$ for some point $c \in C$ whose coordinates are both divisible by four.

(ii) is obviously true if the predecessor and the successor of $z$ in $P$ are the same point; in this case the short path has length 0 . In the other cases, (ii) can be verified by inspection of Fig. 7: In view of the symmetries of the connectedness graph of $w$, we may assume, without loss of generality, that the point $z$ lies in $h^{-1}(\{(2,2)\})=\bar{A}_{8}((4,4))$, where $(2,2) \in D$ (so that $(4,4) \in C$ ) and, since $z \neq(4,4)$ (as $\left.z \in C_{i}^{\prime \prime}\right)$, we may further assume that

either (A) $z=(4,5)$, and the predecessor and the successor of $z \in P$ are $(4,6)$ and $(5,6)$ or vice versa; or (B) $z=(4,5)$, and the predecessor and the successor of $z \in P$ are $(3,6)$ and $(5,6)$;

or (C) $z=(5,5)$, and the predecessor and the successor of $z \in P$ are $(5,6)$ and $(6,6)$ or vice versa;

or (D) $z=(5,5)$, and the predecessor and the successor of $z \in P$ are $(5,6)$ and $(6,5)$.

In case (A), (ii) holds because $(4,6)$ is adjacent to $(5,6)$. In cases $(B)$ and $(C)$, we first note that $(4,6) \notin$ $h^{-1}(D)$ - for otherwise $\{(3,6),(4,6),(5,6)\}=h^{-1}(\{(2,3)\}) \subseteq D_{0}$ and so not all points of $P$ are in 

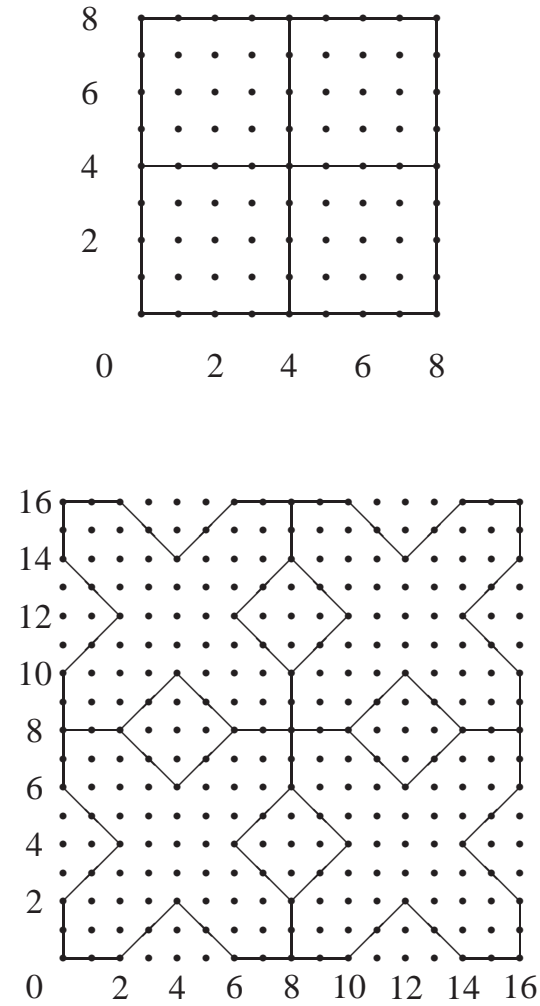
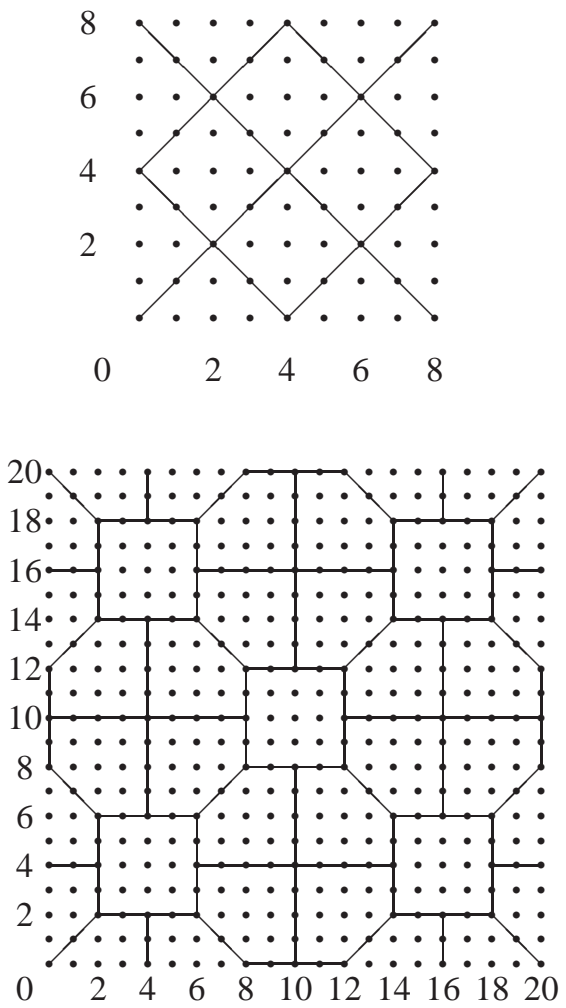

Fig. 8: Portions of four graphs on $\mathbb{Z}^{2}$.

$C_{i}^{\prime \prime}$, which is a contradiction. Thus, in case (B), the path whose points are $(3,6),(4,6)$, and $(5,6)$ satisfies the condition of (ii). In case (C), since $(4,6) \notin h^{-1}(D)$, we have that $(4,8) \notin C$ (by condition (a) in the first paragraph of this proof), which implies $(5,7) \notin h^{-1}(D)$; so the path whose points are $(5,6)$, $(5,7)$, and $(6,6)$, or its reverse, satisfies the condition of (ii). In case (D), we can similarly deduce that $(5,7) \notin h^{-1}(D)$ and $(7,5) \notin h^{-1}(D)$; moreover, $(6,6) \notin C$ (as $(6,6)$ cannot be adjacent to two points of the simple closed curve $C$ in the connectedness graph of $w$ because $(5,7) \notin C,(7,5) \notin C$, and $(5,5)=z \notin C)$, and so $(6,6) \notin h^{-1}(D)$. Thus, in case (D), the path whose points are $(5,6),(5,7),(6,6)$, $(7,5)$, and $(6,5)$ satisfies the condition of (ii).

Example 4.3 Every cycle in any of the four graphs portions of which are shown in Figure 8 is a Jordan curve in $\left(\mathbb{Z}^{2}, v\right)$.

\section{Concluding remarks}

Jordan curves play an important role in computer image processing because they represent boundaries of regions of digital images. It may therefore be useful to deal with topologies providing a rich enough 
variety of Jordan curves in the digital plane for the study of digital images. One of the topologies with this property is the well-known Khalimsky topology. In this note, we have introduced two more such topologies, namely $w$ and $v$. The results obtained suggest that these topologies might be used as background structures on the digital plane to solve problems of digital image processing, especially those closely related to boundaries (image data compression, pattern recognition, boundary detection and contour filling, etc.). The topology $w$ has an advantage over the Khalimsky topology consisting in the fact that Jordan curves in $\left(\mathbb{Z}^{2}, w\right)$ may turn, at some points, to make an acute angle $\frac{\pi}{4}$.

\section{Acknowledgements}

The author would like to thank the anonymous referees for their remarks and suggestions, which helped to improve the paper substantially.

\section{References}

[1] V. Capraro. A notion of continuity in discrete spaces and applications. To appear in Appl. Gen. Topol, http://arxiv.org/abs/1210.2352.

[2] E. Čech. Topological Spaces (Revised by Z.Frolik and M.Katětov). Academia, Prague, 1966.

[3] U. Eckhardt and L.J. Latecki. Topologies for the digital spaces $\mathbb{Z}^{2}$ and $\mathbb{Z}^{3}$. Comput. Vision Image Understanding, 90:295-312, 2003.

[4] R. Engelking. General Topology. Państwowe Wydawnictwo Naukowe, Warszawa, 1977.

[5] E.D. Khalimsky. On topologies of generalized segments. Soviet Math. Dokl., 10:1508-1511, 1999.

[6] E.D. Khalimsky, R. Kopperman and P.R. Meyer. Computer graphics and connected topologies on finite ordered sets. Topology Appl., 36:1-17, 1990.

[7] E.D. Khalimsky, R. Kopperman and P.R. Meyer. Boundaries in digital planes. Jour. of Appl. Math. and Stoch. Anal., 3:27-55, 1990.

[8] C.O. Kiselman. Digital Jordan curve theorems. Lect. Notes in Comp. Sci., 1953:46-56, 2000.

[9] T.Y. Kong, R. Kopperman and P.R. Meyer. A topological approach to digital topology. Amer. Math. Monthly, 98:902-917, 1991.

[10] R. Kopperman, P.R. Meyer and R.G. Wilson. A Jordan surface theorem for three-dimensional digital spaces. Discr. and Comput. Geom., 6:155-161, 1991.

[11] D. Marcus at al. A special topology for the integers (Problem 5712). Amer. Math. Monthly, 77:1119, 1970.

[12] A. Rosenfeld. Digital topology. Amer. Math. Monthly, 86:621-630, 1979.

[13] A. Rosenfeld. Picture Languages. Academic Press, New York, 1979.

[14] J. Šlapal. Closure operations for digital topology. Theor. Comp. Sci., 305:457-471, 2003.

[15] J. Šlapal. A digital analogue of the Jordan curve theorem. Discr. Appl. Math., 139:231-251, 2004.

[16] J. Šlapal. Digital Jordan curves. Top. Appl., 153:3255-3264, 2006.

[17] J. Šlapal. A quotient-universal digital topology. Theor. Comp. Sci., 405:164-175, 2008.

[18] J. Šlapal. Convenient closure operators on $\mathbb{Z}^{2}$. Lect. Notes in Comp. Sci. 5852:425-436, 2009.

[19] J. Šlapal. A Jordan curve theorem in the digital plane. Lect. Notes in Comp. Sci., 6636:120-131, 2009. 